\title{
A Novel Design of Sparse FIR Multiple Notch Filters with Tunable Notch Frequencies
}

\author{
Wei Xu, ${ }^{1,2}$ Anyu Li $\mathbb{D}^{1,2}$ Boya Shi, ${ }^{1,2}$ and Jiaxiang Zhao $\mathbb{D}^{3}$ \\ ${ }^{1}$ School of Electronics and Information Engineering, Tianjin Polytechnic University, Tianjin 300387, China \\ ${ }^{2}$ Tianjin Key Laboratory of Optoelectronic Detection Technology and Systems, Tianjin 300387, China \\ ${ }^{3}$ College of Electronic Information and Optical Engineering, Nankai University, Tianjin 300071, China
}

Correspondence should be addressed to Jiaxiang Zhao; zhaojx@nankai.edu.cn

Received 27 December 2017; Revised 14 March 2018; Accepted 2 April 2018; Published 8 May 2018

Academic Editor: Eric Feulvarch

Copyright (C) 2018 Wei Xu et al. This is an open access article distributed under the Creative Commons Attribution License, which permits unrestricted use, distribution, and reproduction in any medium, provided the original work is properly cited.

\begin{abstract}
We focus on the design of finite impulse response (FIR) multiple notch filters. To reduce the computational complexity and hardware implementation complexity, a novel algorithm is developed based on the mixture of the tuning of notch frequencies and the sparsity of filter coefficients. The proposed design procedure can be carried out as follow: first, since sparse FIR filters have lower implementation complexity than full filters, a sparse linear phase FIR single notch filter with the given rejection bandwidth and passband attenuation is designed. Second, a tuning procedure is applied to the computed sparse filter to produce the desired sparse linear phase FIR multiple notch filter. When the notch frequencies are varied, the same tuning procedure can be employed to render the new multiple notch filter instead of designing the filter from scratch. The effectiveness of the proposed algorithm is demonstrated through three design examples.
\end{abstract}

\section{Introduction}

The multiple notch filters, which can highly attenuate some frequency components in the input signal while leaving the others relatively unchanged, are widely used in many applications. Important examples include radar systems, control and instrumentation systems, communications systems, medical applications, biomedical engineering, and indoor localization $[1,2]$.

Various methods [3-8] have been reported to design FIR multiple notch filters. In general, the multiple notch filters derived from these algorithms are not sparse. Compared with full FIR filters, sparse filters can significantly reduce the implementation complexity in the hardware. In [9], we proposed an iterative reweighed OMP algorithm to compute sparse notch filters. However, when the notch frequencies are varied, it requires one to design the whole filter from scratch, hence increasing the computational complexity of this scheme.

Recently, in [10-12], a number of algorithms are proposed to design FIR filters based on LMS minimization or Monte Carlo methods. The disadvantage of these approaches is the suboptimality in terms of the filter length related to its selectivity. Another disadvantage is that the attenuation at the notch frequency changes during the adaptation process; therefore, a strong attenuation of the disturbing signal at the notch frequency is not guaranteed. Moreover, the actual value of the attenuation at notch frequency is caused by the adaptation process.

In this brief, the design problems of sparse FIR multiple notch filters with tunable notch frequencies are studied. To reduce the computational complexity and the hardware complexity, a novel algorithm is developed based on the mixture of the tuning of notch frequencies and the sparsity of filter coefficients. The sparse FIR multiple notch filters can significantly reduce the number of the adders and multipliers used in the hardware implementation. However, the design of FIR sparse filter always involves iterative procedures and numerical optimization, which results in a high computational complexity for the practice system. The tuning of notch frequencies is a useful operation for the design of FIR multiple notch filter. In the case of variable notch frequencies, the same tuning process is implemented to obtain the new multiple notch filter instead of designing the filter 
from scratch. Therefore, the tuning feature can significantly reduce the computational complexity. We demonstrate the effectiveness of this approach through three design examples.

\section{Problem Formulation}

Given the design parameters of linear phase FIR multiple notch filter, which include a set of the notch frequencies $\left\{\bar{\omega}_{i}\right\}_{i=1}^{r}$, rejection bandwidth $\Delta \omega$, and passbands attenuation $\alpha$, the given notch frequencies $\left\{\bar{\omega}_{i}\right\}_{i=1}^{r}$ satisfying $\bar{\omega}_{i}<\bar{\omega}_{i+1}$ for $1 \leq i \leq r$ are allowed to be nonuniformly distributed in the set $[0, \pi]$. The ideal multiple notch filter amplitude response $H_{d}(\omega)$ satisfies

$$
H_{d}(\omega)= \begin{cases}0 & \omega \in \Omega^{0} \\ 1 & \omega \in \Omega^{1},\end{cases}
$$

where $\Omega^{0}$ and $\Omega^{1}$ are, respectively, defined as

$$
\begin{aligned}
& \Omega^{0}=\left\{\omega|| \omega-\bar{\omega}_{i} \mid \leq \frac{\Delta \omega}{2}, 1 \leq i \leq r\right\}, \\
& \Omega^{1}=[0, \pi]-\Omega^{0} .
\end{aligned}
$$

To simplify the presentation, we focus on the design of Type-I linear phase FIR filter $H\left(e^{j \omega}\right)=e^{-j M \omega} H_{0}(\omega)$; that is, the filter order $N=2 M$ is even and $h(m)=h(N-m)$ for all $0 \leq m \leq N$. For other types of filter, our design method presented in this letter is feasible. For the case of Type-I filter, the zero-phase amplitude response $H_{0}(\omega)$ can be expressed as

$$
H_{0}(\omega)=h(M)+2 \sum_{m=1}^{M} h(M-m) \cos (m \omega),
$$

with $M=N / 2$.

\section{The Proposed Sparse Linear Phase FIR Multiple Notch Filter Design}

In this section, a novel design method is presented to produce the sparse FIR multiple notch filter. The procedure of computing the linear phase FIR multiple notch filter starts with the estimation of the initial order $N$ of the filter $F\left(e^{j \omega}\right)$ through

$$
N=\max _{i \in\{1, \ldots, r\}} N_{i} .
$$

From [13, eq. (20)], $N_{i}$ is computed as

$$
N_{i}=\max \left\{\widehat{N}\left(\omega_{p_{1 i}}, \Delta F, \delta_{p}, \delta_{s}\right), \widehat{N}\left(\omega_{p_{2 i}}, \Delta F, \delta_{p}, \delta_{s}\right)\right\},
$$

where $\Delta F=\Delta \omega / 2$ and the function $\widehat{N}(\cdot)$ is determined by [13, eq. (15)]. The arguments of $\widehat{N}(\cdot)$ can be computed as

$$
\begin{gathered}
\omega_{p_{1 i}}=\frac{\bar{\omega}_{i}-\Delta F}{2}, \\
\omega_{p_{2 i}}=\frac{1-\bar{\omega}_{i}-\Delta F}{2}, \\
\delta_{p}=\delta_{s}=\frac{1-\alpha}{1+\alpha} .
\end{gathered}
$$

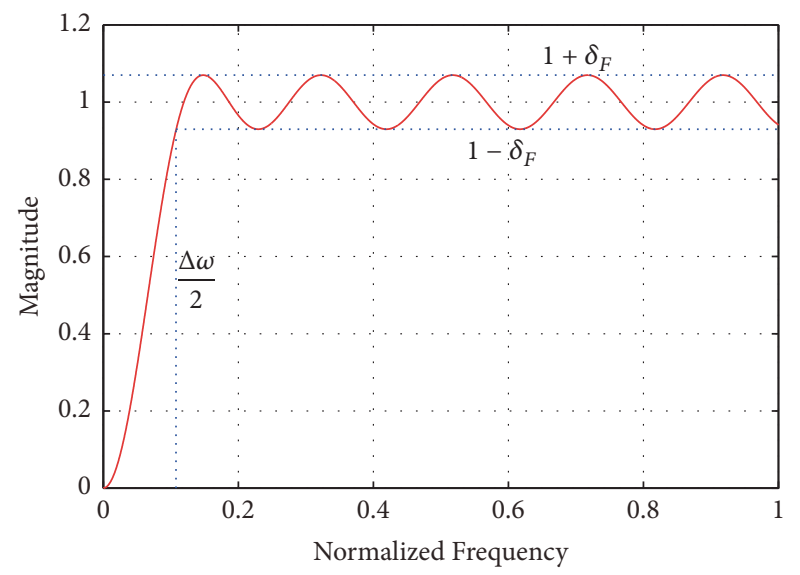

FIGURE 1: The illustration for the amplitude response of the desired sparse single notch filter.

The following design procedure is mainly comprised of two stages: in the first stage, a sparse linear phase FIR single notch filter $F\left(e^{j \omega}\right)$ with the given rejection bandwidth and passband attenuation is designed as a fixed sparse filter. In the next stage, a tuning process is carried out to compute the desired multiple notch filter with the given notch frequencies based on the filter $F\left(e^{j \omega}\right)$.

3.1. Sparse Linear Phase FIR Single Notch Filter Design. In this section, a sparse linear phase FIR single notch filter $F\left(e^{j \omega}\right)$ of order $N$ with the notch frequency $\bar{\omega}_{1}=0$ is designed. Let $F\left(e^{j \omega}\right)=e^{-j M \omega} F_{0}(\omega)$ represent the single notch filter, as shown in Figure 1; the real-valued amplitude response $F_{0}(\omega)$ satisfies

$$
\begin{aligned}
F_{0}(\omega) & =0, \quad \omega=0, \\
0 & <F_{0}(\omega)<1-\delta_{F}, \quad 0<|\omega|<\frac{\Delta \omega}{2}, \\
\left|F_{0}(\omega)-1\right| & <\delta_{F}, \quad \frac{\Delta \omega}{2} \leq|\omega| \leq \pi .
\end{aligned}
$$

The passband ripple $\delta_{F}$ of the single notch filter $F\left(e^{j \omega}\right)$ and the attenuation in the passbands $\alpha$ are related through

$$
\delta_{F}=\frac{1-\alpha}{2 r(1+\alpha)} .
$$

Equation (8) is a conservative choice of $\delta_{F}$ which ensures the multiple notch filter yielded from this choice to satisfy the design specifications. In most cases, $\delta_{\mathrm{F}}$ can be chosen between $(1-\alpha) / 2 r(1+\alpha)$ and $(1-\alpha) /(1+\alpha)$.

The design of the sparse single notch filter $F\left(e^{j \omega}\right)$ can be formulated as

$$
\begin{array}{ll}
\min _{\mathbf{f}} & \|\mathbf{f}\|_{0} \\
\text { s.t. } & |\mathbf{c}(\omega) \mathbf{f}-1| \leq \delta_{F}, \quad \omega \in\left[\frac{\Delta \omega}{2}, \pi\right], \\
& \mathbf{c}(\omega) \mathbf{f}=0, \quad \omega=0,
\end{array}
$$


where we have

$$
\begin{aligned}
& \mathbf{c}(\omega)=\left[\begin{array}{llllll}
1 & \cos (\omega) & \cdots & \cos (m \omega) & \cdots & \cos (M \omega)
\end{array}\right] \\
& \mathbf{f}=\left[\begin{array}{llllll}
f(M) & 2 f(M-1) & \cdots & 2 f(m) & \cdots & 2 f(0)
\end{array}\right]^{T},
\end{aligned}
$$

with $0 \leq m \leq M$.

To compute a solution of problem (9a), (9b), and (9c), we follow the standard discretization procedure as presented in [14] and replace the continuous parameter $\omega$ by $L$ samples (where $L \gg 1$ is a large positive integer) uniformly distributed in the frequency set $[\Delta \omega / 2, \pi]$. Thus, the discretization and normalized formulation of problem (9a), (9b), and (9c) is given by

$$
\begin{array}{ll}
\min _{\mathbf{f}} & \|\mathbf{f}\|_{0} \\
\text { s.t. } & \left|\mathbf{A f}-\mathbf{1}_{L \times 1}\right| \leq \delta_{\mathrm{F}} \cdot \mathbf{1}_{L \times 1}, \\
& \mathbf{1}_{1 \times L} \mathbf{f}=0,
\end{array}
$$

where we have

$$
\mathbf{A}=\left[\begin{array}{c}
\mathbf{c}\left(\omega_{1}\right) \\
\mathbf{c}\left(\omega_{2}\right) \\
\vdots \\
\mathbf{c}\left(\omega_{l}\right) \\
\vdots \\
\mathbf{c}\left(\omega_{L}\right)
\end{array}\right]=\left[\begin{array}{cccc}
1 & \cos \left(\omega_{1}\right) & \cdots & \cos \left(M \omega_{1}\right) \\
1 & \cos \left(\omega_{2}\right) & \cdots & \cos \left(M \omega_{2}\right) \\
\vdots & \vdots & \ddots & \vdots \\
1 & \cos \left(\omega_{l}\right) & \cdots & \cos \left(M \omega_{l}\right) \\
\vdots & \vdots & \ddots & \vdots \\
1 & \cos \left(\omega_{L}\right) & \cdots & \cos \left(M \omega_{L}\right)
\end{array}\right],
$$

with $\omega_{l} \in[\Delta \omega / 2, \pi]$ and $1 \leq l \leq L$.

It is known that this optimization problem is in general NP-hard due to the existence of $l_{0}$-norm in its objective function. To tackle this problem, a great deal of effort has been made to develop efficient algorithms. In this paper, we can employ one of these sparse filter algorithms, for example, linear programming [15], iterative second-order cone programming (ISOCP) [16], iterative reweighted $l_{1}$ (IRL1) [17], and iterative reweighted OMP (IROMP) schemes [9], to attain the desired sparse FIR single notch filter.

\subsection{The Design of the Desired Linear Phase FIR Multiple Notch} Filter. In this section, a tuning process is implemented to derive the desired FIR multiple notch filter based on $F\left(e^{j \omega}\right)$ of the previous stage.

For the given notch frequencies set $\left\{\bar{\omega}_{i}\right\}_{i=1}^{r}$, the multiple notch filter $H\left(e^{j \omega}\right)$ can be given as

$$
H\left(e^{j \omega}\right)=e^{-j M \omega} \sum_{i=1}^{r}\left[F_{0}\left(\omega+\bar{\omega}_{i}\right)+F_{0}\left(\omega-\bar{\omega}_{i}\right)\right] .
$$

According to the Fourier transform theory, the impulse response $h(n)$ of $H\left(e^{j \omega}\right)$ can be obtained as

$$
h(n)=\sum_{i=1}^{r} f(n) \cos \left(n \bar{\omega}_{i}\right)
$$

where $0 \leq n \leq N$.
Compute the attenuation $\widehat{\alpha}$ in the passbands of the linear phase FIR multiple notch filter $H\left(e^{j \omega}\right)$ as

$$
\widehat{\alpha}=\frac{\min \left(H_{0}(\omega)\right)}{\max \left(H_{0}(\omega)\right)}, \quad \omega \in \Omega^{1} .
$$

If $\hat{\alpha} \leq \alpha$, then the computed filter $\{h(n)\}_{n=0}^{N}$ is a sparse solution for the given specifications. Otherwise, the following linear program optimization is run to minimize the attenuation in the passbands of the obtained filter:

$$
\begin{array}{ll}
\min _{\mathbf{h}, \mu} & \mu \\
\text { s.t. } & \left|\mathbf{B h}-\mathbf{1}_{L \times 1}\right| \leq(\delta+\mu) \cdot \mathbf{1}_{L \times 1}, \\
& \mathbf{c}\left(\bar{\omega}_{i}\right) \mathbf{h}=0, \quad i=1,2, \ldots, r, \\
& h(n)=0, \quad n \in \mathscr{Z},
\end{array}
$$

where $\mathscr{Z}$ represents the set of indices at which $h(n)=0$ based on (14) and matrix B can be written as

$$
\mathbf{B}=\left[\begin{array}{c}
\mathbf{c}\left(\omega_{1}^{\prime}\right) \\
\mathbf{c}\left(\omega_{2}^{\prime}\right) \\
\vdots \\
\mathbf{c}\left(\omega_{1}^{\prime}\right) \\
\vdots \\
\mathbf{c}\left(\omega_{\mathrm{L}}^{\prime}\right)
\end{array}\right], \quad \omega_{l}^{\prime} \in \Omega^{1}
$$

If the optimal objective value $\mu$ of (16a), (16b), (16c), and (16d) is negative, that is, $\mu \leq 0$, the obtained filter $\mathbf{h}$ is a sparse solution for the given specifications. Otherwise, the sparsity pattern $\mathscr{Z}$ is infeasible to the given specifications of the multiple notch filter; then the largest element is eliminated from $\mathscr{Z}$ and the linear program (16a), (16b), (16c), and (16d) is solved with the new set $\mathscr{Z}$ until $\mu \leq 0$.

When the notch frequencies are changed, the same tuning process is implemented to yield the new multiple notch filter instead of designing the filter from scratch. Figure 2 outlines the main steps of the proposed algorithm.

\section{Simulation}

In this section, we confirm the effectiveness of our multiple notch filter design scheme through three examples.

Example 1. Let us design a multiple notch filter specified by a set of notch frequencies $\{0.25 \pi, 0.49 \pi, 0.78 \pi\}, \alpha=-0.80$ (passbands attenuation), and $\Delta \omega=0.05 \pi$ (the rejection bandwidths).

By substituting the design specifications into [9, eq. (11)], we obtain the initial order $N=174$. In this simulation, we employ the IROMP scheme [9] to design the sparse single notch filter. As shown in Figure 3, the amplitude response of the multiple notch filter is derived by following 


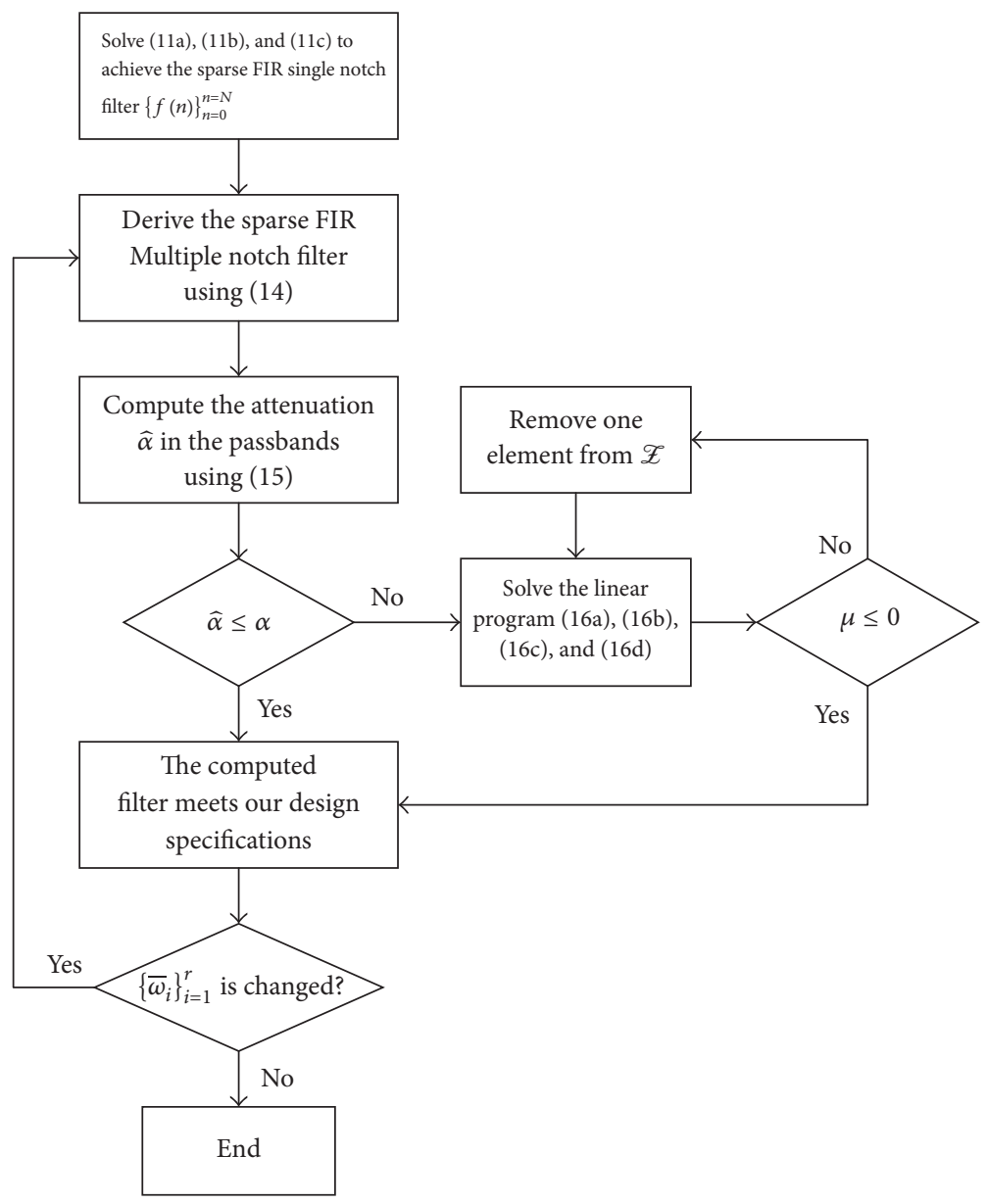

FIGURE 2: Flowchart of the proposed design algorithm.

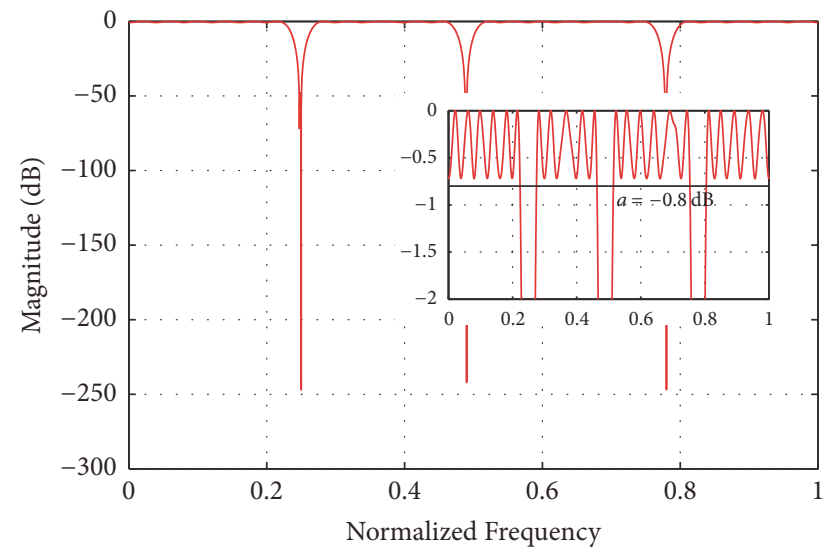

FIGURE 3: The amplitude response of the filter yielded from our design method for Example 1.

steps in Figure 2. It is obvious that the specification is well satisfied. The nonzero tap weights of the multiple notch filter yielded from our design method are listed in Table 1. The filter order, number of nonzero taps, rejection bandwidth, passband attenuation, and attenuation at the notch frequency are listed in Table 2.
Example 2. We only change the notch frequencies from $\{0.25 \pi, 0.49 \pi, 0.78 \pi\}$ of Example 1 to $\{0.34 \pi, 0.43 \pi, 0.72 \pi\}$ but use the same rejection bandwidth and attenuation in the passbands.

Since the same rejection bandwidth and attenuation in the passbands as Example 1 are used, the sparse single notch filter $F\left(e^{j \omega}\right)$ of (1la), (11b), and (11c) with $N=174$ can be identical to the one computed in Example 1. Following the tuning procedure from (14) to (16a), (16b), (16c), and (16d), we compute the sparse multiple notch filter with this new set of the notch frequencies. Figure 4 shows the performance of the sparse multiple notch filter yielded from our scheme. The nonzero tap weights of the multiple notch filter yielded from our design method are listed in Table 3. The filter order, number of nonzero taps, rejection bandwidth, passband attenuation, and attenuation at the notch frequency are listed in Table 2.

Example 3. Change the set of notch frequencies in Example 1 to $\{0.25 \pi, 0.49 \pi, 0.61 \pi, 0.78 \pi\}$, while $\alpha$ and $\Delta \omega$ remain the same.

Since $\alpha$ and $\Delta \omega$ are kept constant, we start with the sparse single notch filter $F\left(e^{j \omega}\right)$ which is the same as that derived in 
TABLE 1: Nonzero coefficients of the designed filter in Example 1.

\begin{tabular}{|c|c|c|}
\hline \multicolumn{2}{|c|}{ Taps } & Nonzero tap weights \\
\hline 0 & 174 & 0.004722848554565 \\
\hline 24 & 150 & 0.002007423266603 \\
\hline 33 & 141 & -0.004871513257007 \\
\hline 38 & 136 & -0.030966811403170 \\
\hline 39 & 135 & -0.022053492615673 \\
\hline 40 & 134 & 0.014951595120278 \\
\hline 41 & 133 & -0.021767842979883 \\
\hline 42 & 132 & 0.011692029570564 \\
\hline 43 & 131 & 0.006950807646344 \\
\hline 44 & 130 & 0.044114166070248 \\
\hline 45 & 129 & 0.006311408033158 \\
\hline 46 & 128 & -0.062066578840798 \\
\hline 47 & 127 & -0.005818521570352 \\
\hline 48 & 126 & -0.002318412822056 \\
\hline 49 & 125 & 0.005091217785500 \\
\hline 50 & 124 & 0.020608511742342 \\
\hline 51 & 123 & -0.014404626498885 \\
\hline 52 & 122 & 0.061394774774335 \\
\hline 53 & 121 & 0.008629427173138 \\
\hline 54 & 120 & -0.072558769634137 \\
\hline 55 & 119 & -0.010787517932644 \\
\hline 56 & 118 & -0.022623175477306 \\
\hline 57 & 117 & 0.030869196951431 \\
\hline 58 & 116 & 0.009932536096148 \\
\hline 59 & 115 & -0.014074814764124 \\
\hline 60 & 114 & 0.094137780017783 \\
\hline 61 & 113 & -0.004199271003407 \\
\hline 62 & 112 & -0.061953987746997 \\
\hline 63 & 111 & -0.047952288148680 \\
\hline 64 & 110 & -0.038531961471284 \\
\hline 65 & 109 & 0.076026372592104 \\
\hline 66 & 108 & -0.015111106082247 \\
\hline 67 & 107 & 0.000183867057454 \\
\hline 68 & 106 & 0.099331267386019 \\
\hline 69 & 105 & -0.012255378374269 \\
\hline 70 & 104 & -0.028055658481912 \\
\hline 71 & 103 & -0.100962624763570 \\
\hline 72 & 102 & -0.038372777955426 \\
\hline 73 & 101 & 0.098683027527897 \\
\hline 74 & 100 & -0.036089958346951 \\
\hline 75 & 99 & 0.029655283702946 \\
\hline 76 & 98 & 0.071889586307707 \\
\hline 77 & 97 & 0.004739347900282 \\
\hline 78 & 96 & -0.002403828886942 \\
\hline 79 & 95 & -0.153598792283481 \\
\hline 80 & 94 & -0.019548891523249 \\
\hline 81 & 93 & 0.089075079456139 \\
\hline 82 & 92 & -0.023403440116641 \\
\hline 83 & 91 & 0.056274737680820 \\
\hline 84 & 90 & 0.018813444978966 \\
\hline 85 & 89 & 0.047899881207932 \\
\hline 86 & 88 & 0.000409323536052 \\
\hline 87 & & 0.911070614139502 \\
\hline
\end{tabular}

Example $1(N=174)$. The sparse multiple notch filter with this new notch frequencies is obtained through the tuning process from (14) to (16a), (16b), (16c), and (16d). Figure 5 illustrates the amplitude response of this filter. It is evident that the specification is satisfied. The nonzero tap weights of the multiple notch filter yielded from our design method are listed in Table 4. The filter order, number of nonzero taps,

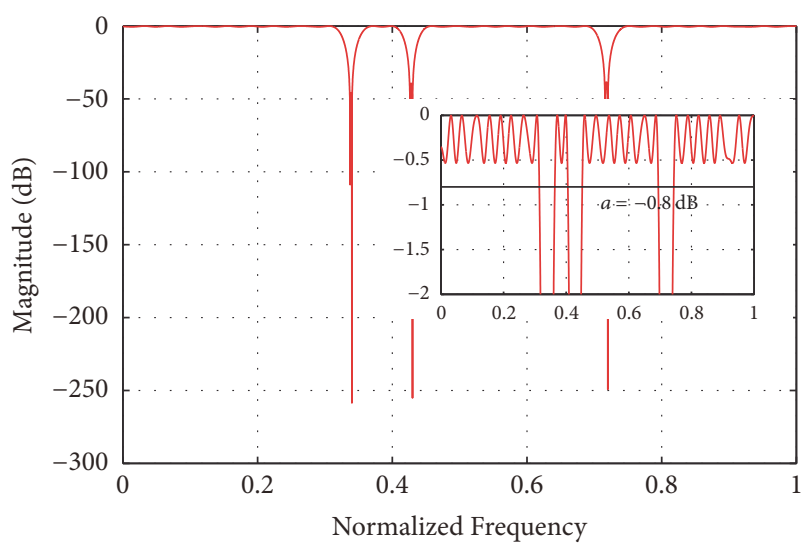

FIGURE 4: The amplitude response of the filter yielded from our design method for Example 2.

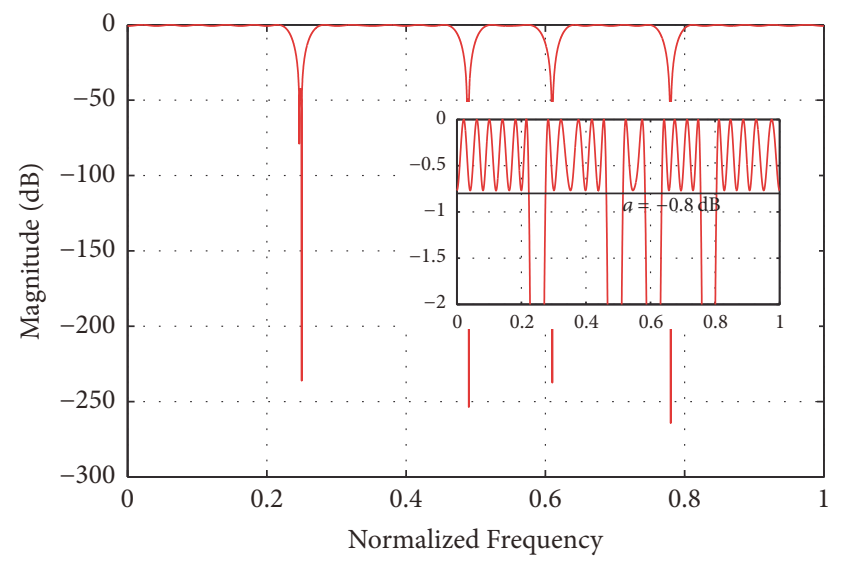

FIGURE 5: The amplitude response of the filter yielded from our design method for Example 3.

rejection bandwidth, passband attenuation, and attenuation at the notch frequency are listed in Table 2.

\section{Conclusion}

In this paper, a novel approach has been presented for the design of sparse FIR multiple notch filters with tunable notch frequencies. To further improve the efficiency, the proposed algorithm is based on the mixture of the tuning of notch frequencies and the sparsity of filter coefficients. In the case of variable notch frequencies, the same tuning procedure can be used to render the new multiple notch filter instead of designing the filter from scratch. Therefore, the proposed algorithm can significantly reduce the computational complexity. Three examples are given to show the effectiveness of this approach.

\section{Conflicts of Interest}

The authors declare that there are no conflicts of interest regarding the publication of this article. 
TABLE 2: A list of filter order, rejection bandwidth, and attenuation of Examples 1-3.

\begin{tabular}{lccccc}
\hline Example & Filter order & $\begin{array}{c}\text { The number of nonzero tap } \\
\text { weights }\end{array}$ & Rejection bandwidth & Passband attenuation & $\begin{array}{c}\text { Attenuation at the notch } \\
\text { frequency }\end{array}$ \\
\hline 1 & 174 & 105 & $0.050 \pi$ & $-0.7197 \mathrm{~dB}$ & $-247 \mathrm{~dB}$ \\
2 & 174 & 105 & $0.050 \pi$ & $-0.5349 \mathrm{~dB}$ & $-259 \mathrm{~dB}$ \\
3 & 174 & 105 & $0.050 \pi$ & $-0.7687 \mathrm{~dB}$ & $-264 \mathrm{~dB}$ \\
\hline
\end{tabular}

TABLE 3: Nonzero coefficients of the designed filter in Example 2.

\begin{tabular}{|c|c|c|}
\hline \multicolumn{2}{|c|}{ Taps } & Nonzero tap weights \\
\hline 0 & 174 & -0.001484391069741 \\
\hline 24 & 150 & 0.015053216345025 \\
\hline 33 & 141 & 0.011203465180753 \\
\hline 38 & 136 & 0.031930539223127 \\
\hline 39 & 135 & -0.011989478832635 \\
\hline 40 & 134 & -0.047916512343373 \\
\hline 41 & 133 & 0.001724422497417 \\
\hline 42 & 132 & 0.012946318823095 \\
\hline 43 & 131 & 0.029367872190180 \\
\hline 44 & 130 & 0.012950777492872 \\
\hline 45 & 129 & -0.058861258880195 \\
\hline 46 & 128 & -0.027161496236364 \\
\hline 47 & 127 & 0.038124403599415 \\
\hline 48 & 126 & 0.005580972074984 \\
\hline 49 & 125 & 0.024077586822642 \\
\hline 50 & 124 & -0.013761958746995 \\
\hline 51 & 123 & -0.036380554634187 \\
\hline 52 & 122 & 0.026803049613006 \\
\hline 53 & 121 & -0.001700050810428 \\
\hline 54 & 120 & -0.023056804511978 \\
\hline 55 & 119 & 0.041926654928440 \\
\hline 56 & 118 & 0.000428542187068 \\
\hline 57 & 117 & -0.005253841608839 \\
\hline 58 & 116 & -0.008896975496026 \\
\hline 59 & 115 & -0.071272465825430 \\
\hline 60 & 114 & 0.037660569869951 \\
\hline 61 & 113 & 0.085639588936268 \\
\hline 62 & 112 & -0.020291841316044 \\
\hline 63 & 111 & -0.035719226300918 \\
\hline 64 & 110 & -0.069237101344906 \\
\hline 65 & 109 & -0.019718488202998 \\
\hline 66 & 108 & 0.128901758725893 \\
\hline 67 & 107 & 0.025726032859600 \\
\hline 68 & 106 & -0.073722260607230 \\
\hline 69 & 105 & -0.025836288834871 \\
\hline 70 & 104 & -0.045891876538100 \\
\hline 71 & 103 & 0.057310758857846 \\
\hline 72 & 102 & 0.075489239705611 \\
\hline 73 & 101 & -0.063149359304339 \\
\hline 74 & 100 & -0.003347070890743 \\
\hline 75 & 99 & 0.014446702737718 \\
\hline 76 & 98 & -0.058103382956618 \\
\hline 77 & 97 & 0.032069195768895 \\
\hline 78 & 96 & 0.000558233096476 \\
\hline 79 & 95 & 0.006719715851877 \\
\hline 80 & 94 & 0.084935075668951 \\
\hline 81 & 93 & -0.076509802170425 \\
\hline 82 & 92 & -0.097984593409281 \\
\hline 83 & 91 & 0.043634372404206 \\
\hline 84 & 90 & 0.040410785159360 \\
\hline 85 & 89 & 0.089360899339001 \\
\hline 86 & 88 & -0.003266951955140 \\
\hline 87 & & 0.915171992267534 \\
\hline
\end{tabular}

TABLE 4: Nonzero coefficients of the designed filter in Example 3.

\begin{tabular}{|c|c|c|}
\hline \multicolumn{2}{|c|}{ Taps } & Nonzero tap weights \\
\hline 0 & 174 & 0.001163545765695 \\
\hline 24 & 150 & 0.003165433288996 \\
\hline 33 & 141 & 0.002091982284630 \\
\hline 38 & 136 & -0.047102411486321 \\
\hline 39 & 135 & -0.005481276840672 \\
\hline 40 & 134 & 0.019772136963283 \\
\hline 41 & 133 & -0.043110955540852 \\
\hline 42 & 132 & 0.020979982565140 \\
\hline 43 & 131 & 0.015127277492607 \\
\hline 44 & 130 & 0.022611789510971 \\
\hline 45 & 129 & 0.010481611606251 \\
\hline 46 & 128 & -0.039298064713159 \\
\hline 47 & 127 & -0.024983870407359 \\
\hline 48 & 126 & -0.013239453713585 \\
\hline 49 & 125 & 0.022935249461144 \\
\hline 50 & 124 & 0.027344765658964 \\
\hline 51 & 123 & -0.037957131548364 \\
\hline 52 & 122 & 0.075665876343805 \\
\hline 53 & 121 & 0.024121474369378 \\
\hline 54 & 120 & -0.104718797612301 \\
\hline 55 & 119 & -0.014707635070068 \\
\hline 56 & 118 & 0.016302396989146 \\
\hline 57 & 117 & 0.011567221940061 \\
\hline 58 & 116 & -0.010934081396023 \\
\hline 59 & 115 & 0.019926233327605 \\
\hline 60 & 114 & 0.089656608769556 \\
\hline 61 & 113 & -0.041103476228622 \\
\hline 62 & 112 & -0.033265661189825 \\
\hline 63 & 111 & -0.026560471743885 \\
\hline 64 & 110 & -0.082576601043643 \\
\hline 65 & 109 & 0.085379844129436 \\
\hline 66 & 108 & 0.024951523169648 \\
\hline 67 & 107 & -0.038897009099235 \\
\hline 68 & 106 & 0.088237797320545 \\
\hline 69 & 105 & 0.036518046705842 \\
\hline 70 & 104 & -0.053707639239860 \\
\hline 71 & 103 & -0.134082248075127 \\
\hline 72 & 102 & 0.007838779814640 \\
\hline 73 & 101 & 0.104434475095505 \\
\hline 74 & 100 & -0.081493323669535 \\
\hline 75 & 99 & 0.057503261219777 \\
\hline 76 & 98 & 0.103032966355301 \\
\hline 77 & 97 & -0.046232816359952 \\
\hline 78 & 96 & -0.001798639004124 \\
\hline 79 & 95 & -0.100284039691277 \\
\hline 80 & 94 & -0.057999870693463 \\
\hline 81 & 93 & 0.060896839992225 \\
\hline 82 & 92 & 0.036785941586311 \\
\hline 83 & 91 & 0.041803167312136 \\
\hline 84 & 90 & -0.030010449210885 \\
\hline 85 & 89 & 0.094188919109719 \\
\hline 86 & 88 & 0.018630470290357 \\
\hline 87 & & 0.882383969239141 \\
\hline
\end{tabular}




\section{Acknowledgments}

This research was supported by the National Natural Science Foundation of China (Grants nos. 61501324 and 61601323) and the Natural Science Foundation of Tianjin (Grant no. 16JCTPJC46900).

\section{References}

[1] M. Vlcek and P. Zahradnik, "Digital multiple notch filters performance," Proceedings of the 15th European Conference on Circuit Theory and Design, pp. 49-52, 2001.

[2] C. K. Ahn, P. Shi, and M. V. Basin, "Deadbeat dissipative FIR filtering," IEEE Transactions on Circuits and Systems I: Regular Papers, vol. 63, no. 8, pp. 1210-1221, 2016.

[3] C.-C. Tseng and S.-C. Pei, "Design of an equiripple FIR notch filter using a multiple exchange algorithm," Signal Processing, vol. 75, no. 3, pp. 225-237, 1999.

[4] P. Zahradnik and M. Vlcek, "Fast Analytical Design Algorithms for FIR Notch Filters," IEEE Transactions on Circuits and Systems I: Regular Papers, vol. 51, no. 3, pp. 608-623, 2004.

[5] P. Zahradnik and M. Vlcek, "An analytical procedure for critical frequency tuning of FIR filters," IEEE Transactions on Circuits and Systems II: Express Briefs, vol. 53, no. 1, pp. 72-76, 2006.

[6] P. Zahradnik and M. Vlcek, "Note on the design of an equiripple DC-notch FIR filter," IEEE Transactions on Circuits and Systems II:Express Briefs, vol. 54, no. 2, pp. 196-199, 2007.

[7] P. Zahradnik, M. Vlcek, and R. Unbehauen, "Design of optimal comb FIR filters-speed and robustness," IEEE Signal Processing Letters, vol. 16, no. 6, pp. 465-468, 2009.

[8] P. Zahradnik and M. Vlček, "Notch filtering suitable for real time removal of power line interference," Radioengineering, vol. 22, no. 1, pp. 186-193, 2013.

[9] W. Xu, J. Zhao, and C. Gu, "Design of linear-phase FIR multiplenotch filters via an iterative reweighted OMP scheme," IEEE Transactions on Circuits and Systems II: Express Briefs, vol. 61, no. 10, pp. 813-817, 2014.

[10] D. O. Olguin, F. Bouchereau, and S. Martinez, "Adaptive notch filter for EEG signals based on the LMS algorithm with variable step-size parameter," Proceedings of the 39th International Conference on Information Sciencesand Systems, 2005.

[11] J. M. Pak, C. K. Ahn, P. Shi, Y. S. Shmaliy, and M. T. Lim, "Distributed hybrid particle/FIR filtering for mitigating NLOS effects in TOA based localization using wireless sensor networks," IEEE Transactions on Industrial Electronics, vol. 64, no. 6, pp. 5182-5191, 2017.

[12] J. M. Pak, C. K. Ahn, Y. S. Shmaliy, P. Shi, and M. T. Lim, "Accurate and Reliable Human Localization Using Composite Particle/FIR Filtering," IEEE Transactions on Human-Machine Systems, vol. 47, no. 3, pp. 332-342, 2016.

[13] K. Ichige, M. Iwaki, and R. Ishii, "Accurate estimation of minimum filter length for optimum FIR digital filters," IEEE Transactions on Circuits and Systems II: Analog and Digital Signal Processing, vol. 47, no. 10, pp. 1008-1016, 2000.

[14] D. Wei, "Non-convex optimization for the design of sparse fir filters," in Proceedings of the 2009 IEEE/SP 15th Workshop on Statistical Signal Processing (SSP), pp. 117-120, Cardiff, UK, August 2009.

[15] T. Baran, D. Wei, and A. V. Oppenheim, "Linear programming algorithms for sparse filter design," IEEE Transactions on Signal Processing, vol. 58, no. 3, part 2, pp. 1605-1617, 2010.
[16] A. Jiang, H. K. Kwan, and Y. Zhu, "Peak-error-constrained sparse FIR filter design using iterative SOCP," IEEE Transactions on Signal Processing, vol. 60, no. 8, pp. 4035-4044, 2012.

[17] C. Rusu and B. Dumitrescu, "Iterative reweighted 11 design of sparse FIR filters," Signal Processing, vol. 92, no. 4, pp. 905-911, 2012. 


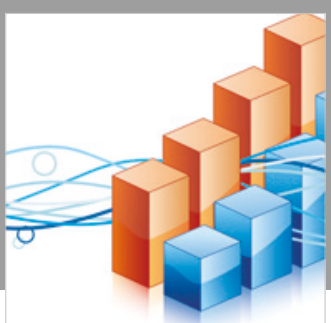

Advances in

Operations Research

\section{-n-m}
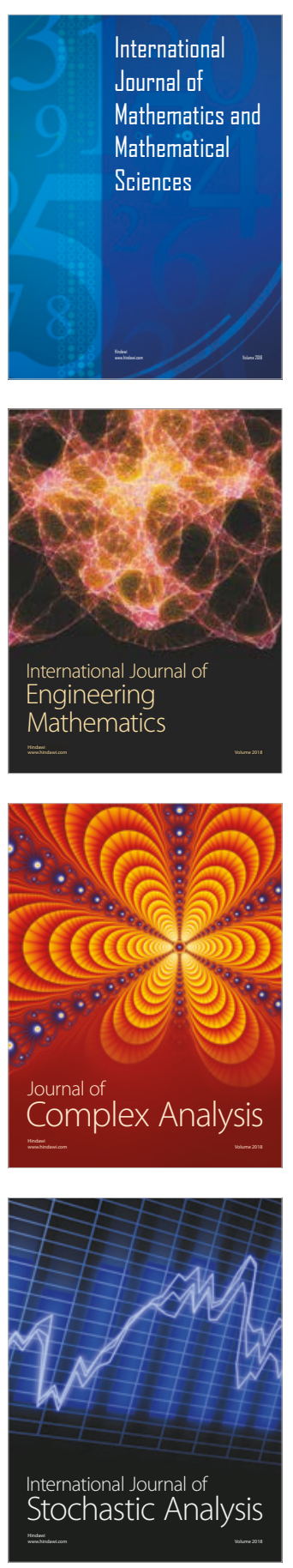
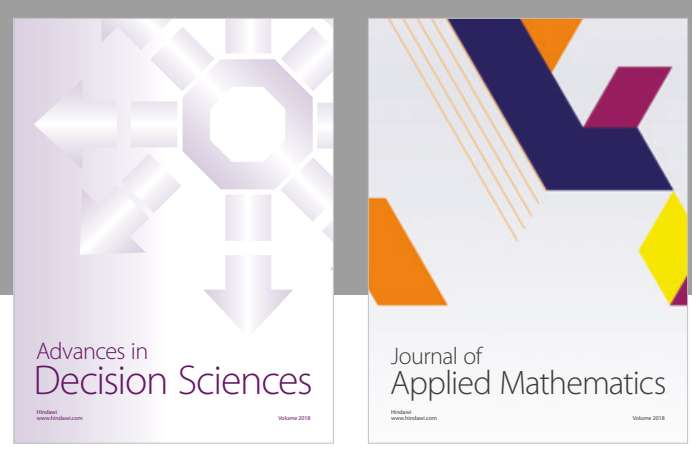

Journal of

Applied Mathematics
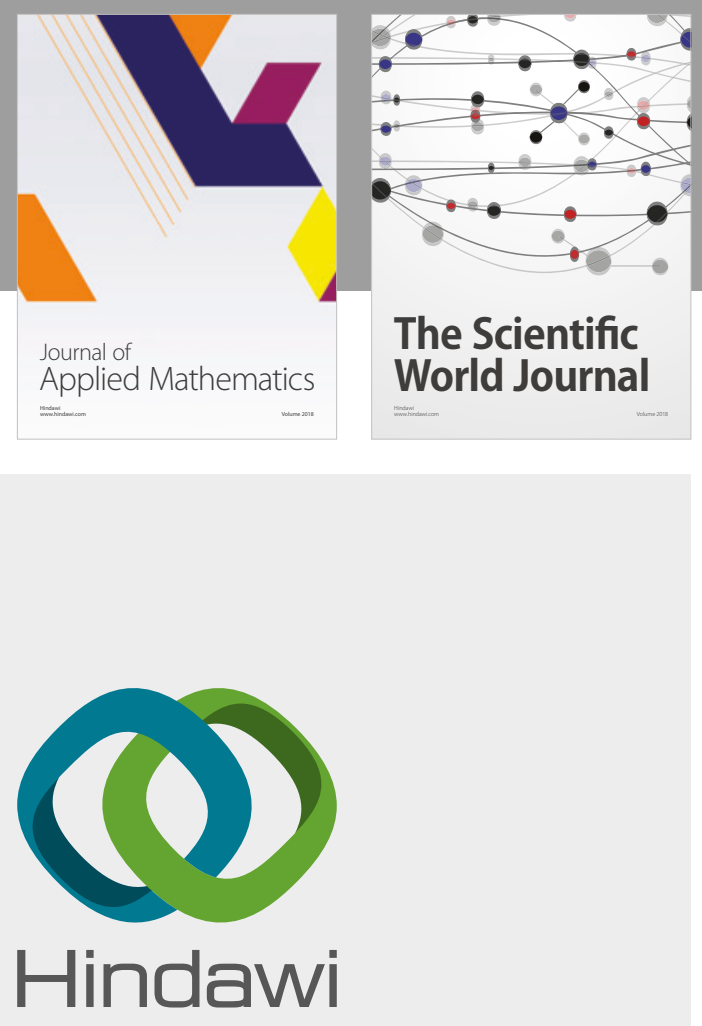

Submit your manuscripts at

www.hindawi.com

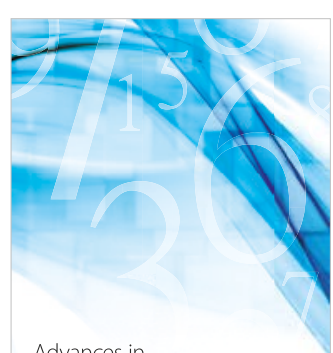

Advances in
Numerical Analysis
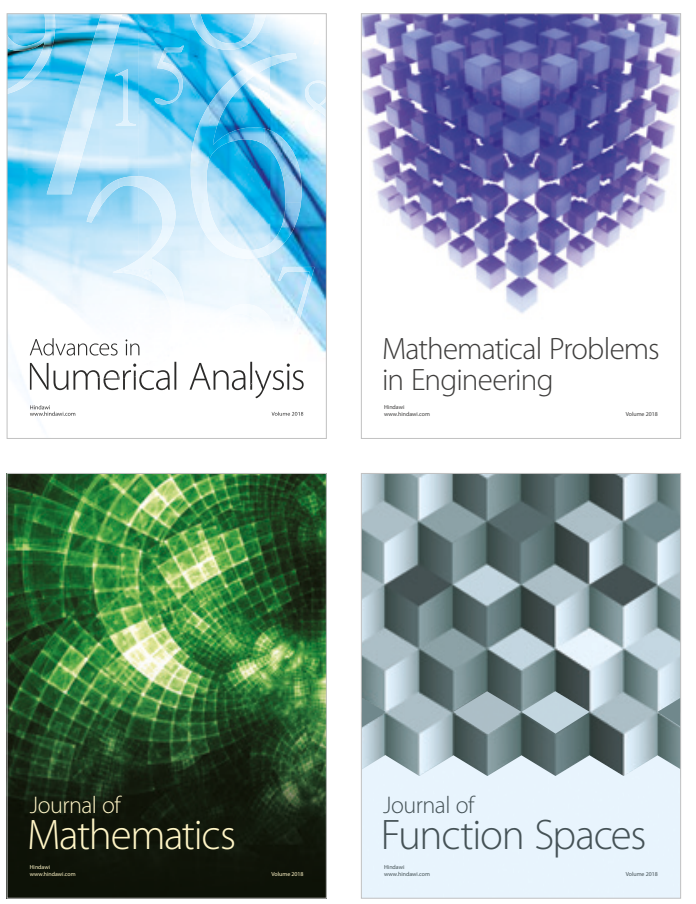

Mathematical Problems in Engineering

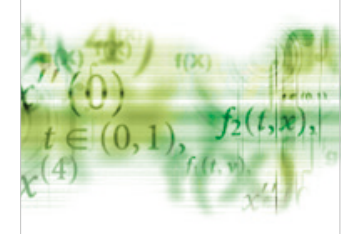

International Journal of

Differential Equations

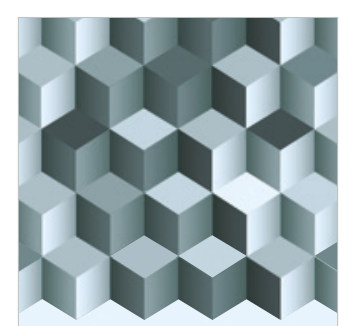

Journal of

Function Spaces

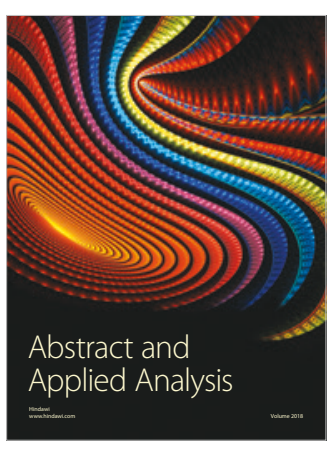

The Scientific

World Journal

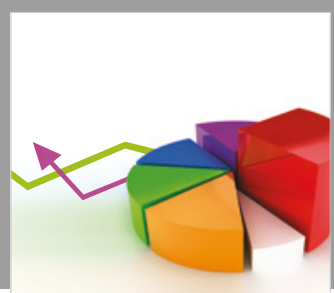

Journal of

Probability and Statistics
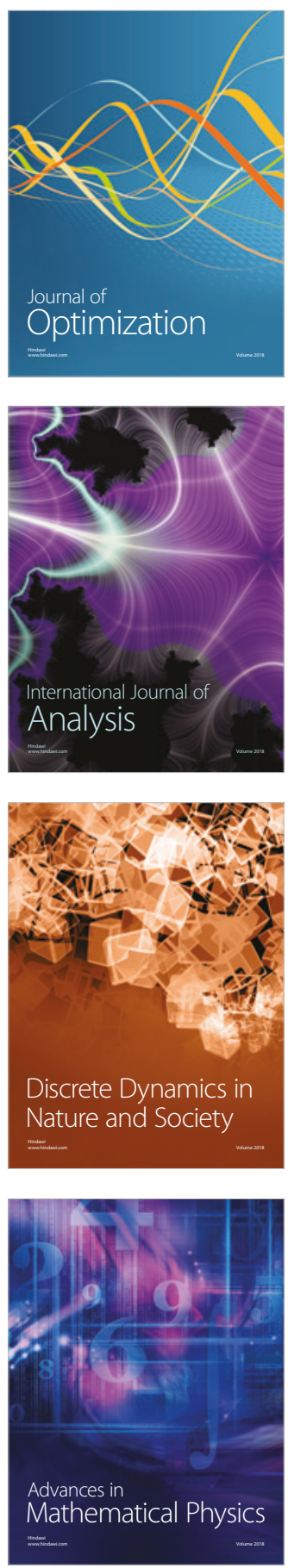